\title{
Femtosecond deep-infrared optical parametric oscillator pumped directly by a Ti:sapphire laser
}

\author{
Callum F. O'Donnell*a, b, S. Chaitanya Kumar, ${ }^{\text {a, b }}$, Kevin T. Zawilski, ${ }^{c}$ Peter G. Schunemann, ${ }^{c}$ and \\ Majid Ebrahim-Zadeh a, b, d
}

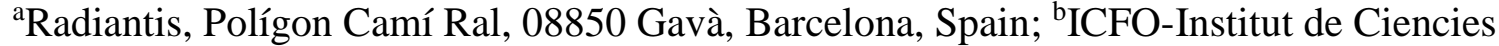 \\ Fotoniques, The Barcelona Institute of Science and Technology, 08860 Castelldefels, Barcelona, \\ Spain; 'BAE Systems, Incorporated, MER15-1813, P.O. Box 868, Nashua, New Hampshire 03061- \\ 0868, USA; 'Institucio Catalana de Recerca i Estudis Avancats (ICREA), Passeig Lluis Companys \\ 23, Barcelona 08010, Spain.
}

\begin{abstract}
We report a high-repetition-rate femtosecond optical parametric oscillator (OPO) for the deep-infrared (deep-IR) based on the nonlinear optical crystal, $\mathrm{CdSiP}_{2}$ (CSP), pumped directly by a Ti:sapphire laser, for the first time. By pumping CSP at $<1 \mu \mathrm{m}$, we have achieved practical output powers at the longest wavelengths generated by any Ti:sapphirepumped OPO. Using a combination of pump wavelength tuning, type-I critical phase-matching, and cavity delay tuning, we have generated continuously tunable radiation across $6654-8373 \mathrm{~nm}\left(1194-1503 \mathrm{~cm}^{-1}\right)$ at $80.5 \mathrm{MHz}$ repetition rate, providing up to $20 \mathrm{~mW}$ of average power at $7314 \mathrm{~nm}$ and $>7 \mathrm{~mW}$ beyond $8000 \mathrm{~nm}$, with idler spectra exhibiting bandwidths of 140-180 nm across the tuning range. Moreover, the near-IR signal is tunable across $1127-1192 \mathrm{~nm}$, providing up to $37 \mathrm{~mW}$ of average power at $1150 \mathrm{~nm}$. Signal pulses, characterised using intensity autocorrelation, have durations of $\sim 260-320 \mathrm{fs}$, with corresponding time-bandwidth product of $\Delta v \Delta \tau \sim 1$. The idler and signal output exhibit a $\mathrm{TEM}_{00}$ spatial profile with single-peak Gaussian distribution. With an equivalent spectral brightness of $\sim 6.68 \times 10^{20}$ photons $\mathrm{s}^{-1} \mathrm{~mm}^{-2} \mathrm{sr}^{-1} 0.1 \% \mathrm{BW}^{-1}$, this OPO represents a viable table-top alternative to synchrotron and supercontinuum sources for deep-IR applications in spectroscopy, metrology and medical diagnostics.
\end{abstract}

Keywords: Optical parametric oscillators, nonlinear optics, ultrafast optics, nonlinear materials, infrared sources.

\section{INTRODUCTION}

High power, high-repetition-rate femtosecond sources of coherent deep-infrared (deep-IR) radiation are in demand for ever increasing range of applications, from vibrational spectroscopy to medical imaging. ${ }^{1}$ In particular, the spectral region of 7-8.5 $\mu \mathrm{m}$ is of great interest due to the presence of strong absorption lines corresponding to the amide III functional group, and various chemical pollutants. ${ }^{2}$ The primary diagnostic technique used to analyse such samples is Fourier Transform Infrared Spectroscopy (FTIR), involving the illumination of a sample using a broadband IR source such as a globar. Although the spectral coverage is broad, the diffuse and incoherent nature of the light source places limitations on the achievable spatial resolution and signal-to-noise ratio. ${ }^{3}$ In the absence of conventional solid-state lasers at these wavelengths, a variety of different approaches towards the development of high-power coherent mid-IR and deep-IR sources have been explored, including supercontinuum generation, synchrotron sources, and quantum cascade lasers (QCLs). ${ }^{4-6}$ However, the use of these technologies is associated with compromises in spectral intensity, available beam time, and tunability, respectively. On the other hand, nonlinear parametric down-conversion techniques can exploit well-established near-IR solid-state and fibre laser technology to generate powerful coherent radiation far into the infrared. ${ }^{7}$ Furthermore, the temporal qualities of the pump laser are transferred to the down-converted beams, enabling high-repetition-rate quasi-continuous-wave pulse trains with $\mathrm{kW}$-level peak power. While commercial OPOs are capable of directly producing hundreds of milliwatts of average power up to and above $\sim 4 \mu \mathrm{m}$ in the mid-IR, efforts to extend their wavelength access into the deep-IR have been hampered by stringent nonlinear material requirements. The critical properties include high nonlinear coefficient, $d_{\text {eff }}$, resistance to damage at high optical intensities, high bulk

*Corresponding author: callum.odonnell@ radiantis.com 
transparency, and ability to be phase-matched at the desired pump, signal and idler wavelengths. The breakthrough finally came by the successful development of cadmium silicon phosphide, $\mathrm{CdSiP}_{2}$ (CSP), a chalgocenide crystal which was first grown to sufficient optical quality in $2010 .{ }^{8}$ With a nominal transparency across $1-7 \mu \mathrm{m}$, thermal conductivity of $13.6 \mathrm{~W} / \mathrm{m} \cdot \mathrm{K}$, and $d_{\text {eff }} \sim 84 \mathrm{pm} / \mathrm{V}$, it enables single-stage conversion from $1.06 \mu \mathrm{m}$ to the mid-IR and deep-IR. ${ }^{9}$ Moreover, its nonlinear figure-of-merit $\left(\mathrm{FOM}=d_{\mathrm{eff}}{ }^{2} / n^{3}\right)$ ranks among the highest of all existing nonlinear crystals. To date, ultrafast OPOs based on CSP have been operated at a fixed angle under noncritical phase-matching (NCPM), with spectral tuning achieved via cavity delay, or by varying the pump wavelength. ${ }^{10-13}$ A summary of previous demonstrations of femtosecond OPOs based on CSP is presented in Table 1. In this work, we add another important milestone to this list, by demonstrating a deep-IR femtosecond OPO based on CSP, pumped directly at by a Kerr-lensmode-locked (KLM) Ti:sapphire laser, for the first time. In doing so, we show that tunable deep-IR radiation can be generated in a single conversion stage using well-established ultrafast pump laser technology.

Table 1: Summary of previous demonstrations of femtosecond OPOs based on CSP.

\begin{tabular}{|c|c|c|c|c|c|c|c|}
\hline Pump & $\begin{array}{l}\text { Rep. rate } \\
(\mathrm{MHz})\end{array}$ & $\begin{array}{l}\text { Tuning } \\
\text { method }^{\mathrm{a}}\end{array}$ & $\begin{array}{l}\text { Idler } \\
(\mu \mathrm{m})^{\mathrm{b}}\end{array}$ & $\begin{array}{l}\text { Pump power }(\mathbf{W}) \\
\text { (wavelength }(\mathrm{nm}) \text { ) }\end{array}$ & $\begin{array}{l}\text { Idler power }(\mathbf{m W}) \\
{\text { (wavelength }(\mu \mathrm{m})^{\mathrm{c}}}^{\text {(w) }}\end{array}$ & $\begin{array}{c}\text { Idler pulse } \\
\text { duration (fs) }\end{array}$ & Ref. \\
\hline Yb:KYW & 100 & - & 6.2 & $1.6(1053)$ & - & - & 10 \\
\hline $\mathrm{Yb}: \mathrm{KGW}$ & 43 & $\mathrm{C}$ & $6.8-7.0$ & $5(1029)$ & $110(7.0)$ & $\sim 500$ (est.) & 11 \\
\hline $\begin{array}{c}\text { Intracavity } \\
\text { MgO:PPLN OPO }\end{array}$ & 76 & $\mathrm{C}$ & $5.9-8.1$ & 0.9 (1024) & $0.064(6.8)$ & - & 12 \\
\hline Commercial OPO & 80 & $\mathrm{P}, \mathrm{C}$ & $6.3-7.0$ & $1(1015-1074)$ & $32(6.8)$ & 200 (est.) & 13 \\
\hline Ti:sapphire & 80 & $\mathrm{P}, \mathrm{A}, \mathrm{C}$ & $6.7-8.4$ & $\mathbf{0 . 8 3}(993-1011)$ & $\begin{array}{c}20(7.3) \\
7(8.1)\end{array}$ & $\sim 300$ (est.) & $\begin{array}{l}\text { This } \\
\text { work }\end{array}$ \\
\hline
\end{tabular}

aTuning methods: C: Cavity delay tuning, P: Pump wavelength tuning, A: Angle tuning.

bIdler range defined relative to weighted centre of most extreme recorded spectra, not $10 \mathrm{~dB}$ limit.

'Peak idler power and power at selected wavelengths of interest.

\section{RESULTS AND DISCUSSION}

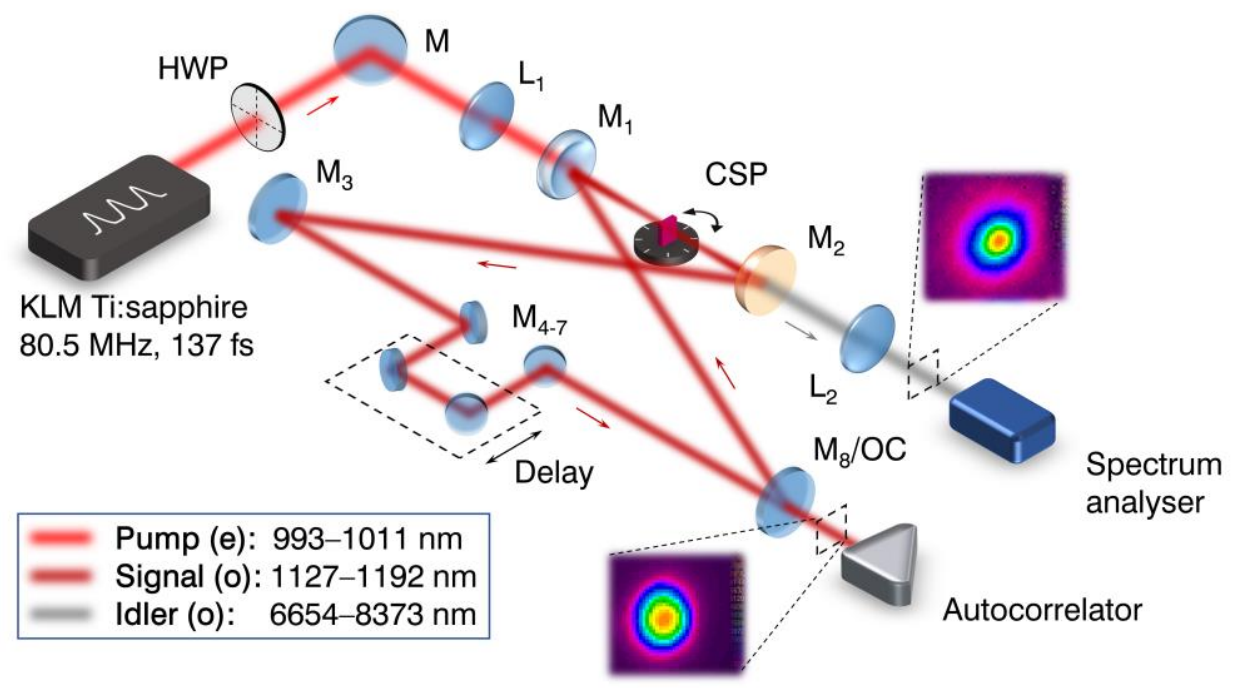

Figure 1. Schematic of the experimental setup for the Ti:sapphire-pumped CSP femtosecond OPO. HWP: Half wave plate, M: Mirrors, OC: Output coupler, L: Lenses, F: Filters. 
The experimental setup for the CSP-based femtosecond OPO is depicted in Fig. 1. The OPO is synchronously pumped by $\Delta \tau \sim 137$ fs input pulses from a KLM Ti:sapphire laser (Spectra-Physics, Mai Tai HP) at a repetition rate of $80.5 \mathrm{MHz}$. For this experiment, to conform with the chosen optical coatings, we operated at pump wavelengths across 993-1011 $\mathrm{nm}$, where the measured FWHM spectral bandwidth was $\Delta \lambda \sim 10.5 \mathrm{~nm}$, corresponding to a time-bandwidth product of $\Delta v \Delta \tau \sim 0.44$, close to the transform limit for $\operatorname{sech}^{2}$ pulses. After transmission through $\mathrm{L}_{1}$ and $\mathrm{M}_{1}$, the average pump power incident on the crystal is $\sim 430-690 \mathrm{~mW}$. The CSP sample is 1 - $\mathrm{mm}$-long with a $4 \mathrm{~mm} \times 5 \mathrm{~mm}$ aperture, and cut at $\theta=90^{\circ}$ $\left(\phi=45^{\circ}\right)$ for type-I $(e \rightarrow o o)$ NCPM at normal incidence. The crystal is maintained at room temperature and mounted on a precision rotation stage, with both end faces antireflection (AR)-coated for high transmission at the pump ( $T>99 \%$ over 950-1080 nm), signal ( $T>99 \%$ across $1100-1200 \mathrm{~nm}$ ), and idler ( $T>93 \%$ across $7000-8500 \mathrm{~nm})$. The pump polarisation is controlled using a half-wave plate $(\lambda / 2)$ and the beam is focused to a waist radius of $w_{0} \sim 28 \mu \mathrm{m}$ at the centre of the crystal using a lens $\left(\mathrm{L}_{1}\right)$ of focal length, $f=100 \mathrm{~mm}$. The OPO cavity comprises two plano-concave mirrors $\left(\mathrm{M}_{1-2}\right)$ with radius of curvature, $\mathrm{r}=100 \mathrm{~mm}$, and six plane mirrors $\left(\mathrm{M}_{3-8}\right)$. $\mathrm{M}_{1}$ transmits the pump (T 83\% at $993 \mathrm{~nm}$ to $T \sim 83 \%$ at $1011 \mathrm{~nm})$ and is highly reflecting (HR) for the signal $(R>99 \%$ across $1100-1300 \mathrm{~nm}) . \mathrm{M}_{2}$ is a 3-mm-thick ZnSe substrate, AR-coated for the deep-IR idler ( $T>94 \%$ across $6500-9000 \mathrm{~nm})$, and HR for the signal $(R>97 \%$ across 1100 $1300 \mathrm{~nm}) . \mathrm{M}_{8}$ is a 5\% signal output coupler $(\mathrm{OC})(\mathrm{R}=95 \%$ across $1100-1300 \mathrm{~nm})$. All remaining mirrors $\left(\mathrm{M}_{3-7}\right)$ are fused silica substrates, HR for the signal $(R>99.8 \%$ across $1100-1300 \mathrm{~nm})$, and negatively chirped for dispersion compensation (GDD -100 - -400 $\mathrm{fs}^{2}$ across $1100-1160 \mathrm{~nm}$ ). The OPO is thus singly-resonant for the near-IR signal wave, with the undepleted pump exiting the cavity through $\mathrm{M}_{8}$, while the single-pass deep-IR idler is transmitted through $\mathrm{M}_{2}$. Finally, after appropriate filtering, the idler is collimated by a $\mathrm{CaF}_{2}$ lens with good deep-IR transmission $(T>60 \%$ over $6000-8500 \mathrm{~nm})$.
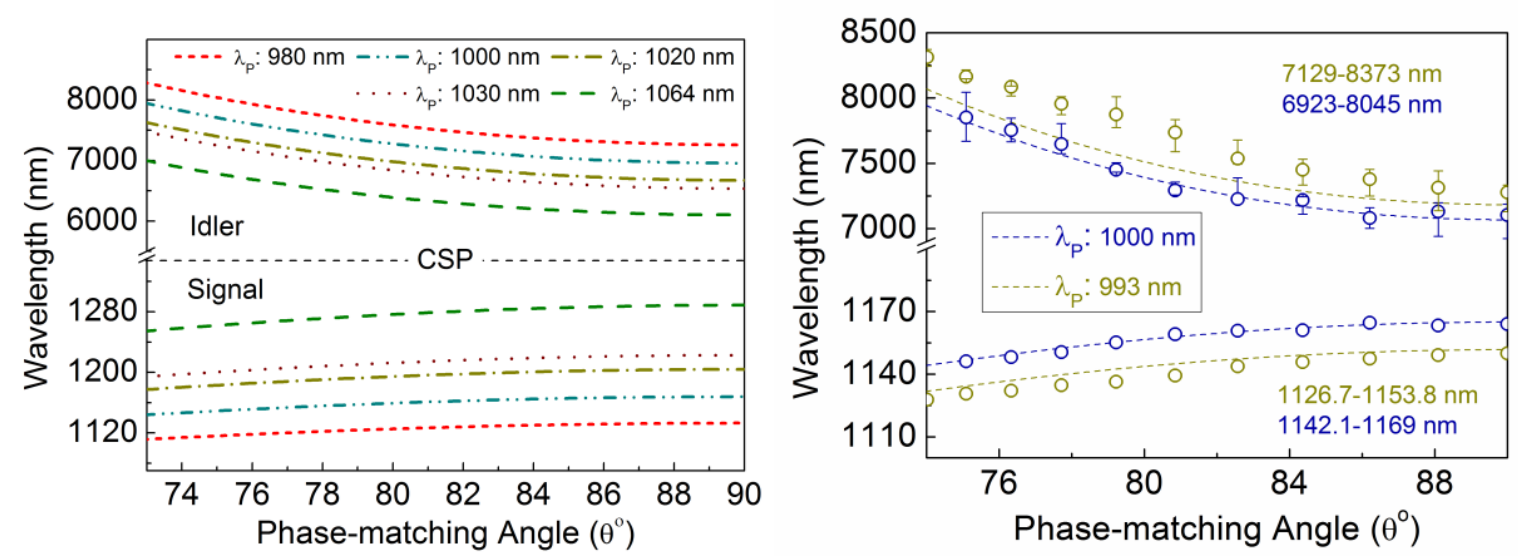

Figure 2. a) Signal and idler tuning curves for type-I $(e \rightarrow o o)$ critical phase-matching as a function of the CSP internal angle for various pump wavelengths in the range so far used to pump CSP OPOs, b) Idler and signal tuning as a function of the internal phase-matching angle in the CSP crystal, for pump wavelengths of $993 \mathrm{~nm}$ and $1000 \mathrm{~nm}$, compared to the wavelength tuning curves predicted using the Sellmeier equations ${ }^{8}$. Error bars represent additional cavity delay tuning.

Operation of the OPO was achieved by assembling the cavity as shown in Fig. 1, and synchronisation was obtained by fine adjustment of the delay line. Deployment of the KLM Ti:sapphire laser offers the important advantage of pump wavelengths $<1 \mu \mathrm{m}$, which enables the generation of longer idler wavelengths in the deep-IR. This is illustrated in Fig. 2(a), where the signal and idler wavelength tuning as function of the CSP phase-matching angle under critical type-I $(e \rightarrow o o)$ phase-matching for various discrete pump wavelengths of interest, from Yb-based solid-state and fibre lasers down to the Ti:sapphire laser, is presented. As can be clearly seen, for all phase-matching angles (including $\theta=90^{\circ}$ at NCPM), longer deep-IR idler wavelengths can be generated by deploying shorter pump wavelengths in the Ti:sapphire laser wavelength range. The results of angle tuning from $\theta=90^{\circ}$ to $74^{\circ}$ are shown in Fig. 2(b), where it can be seen that the near-IR signal wavelength could be tuned across $1126.7-1153.8 \mathrm{~nm}$, with the idler covering a spectral range of 6923-8373 $\mathrm{nm}$ in the deep-IR, for two pump wavelengths at $993 \mathrm{~nm}$ and $1000 \mathrm{~nm}$. The measured values are also compared to the Sellmeier predictions, ${ }^{8}$ with the observed offset accredited to strong self-phase modulation (SPM) redistributing the spectral density towards shorter wavelengths. 

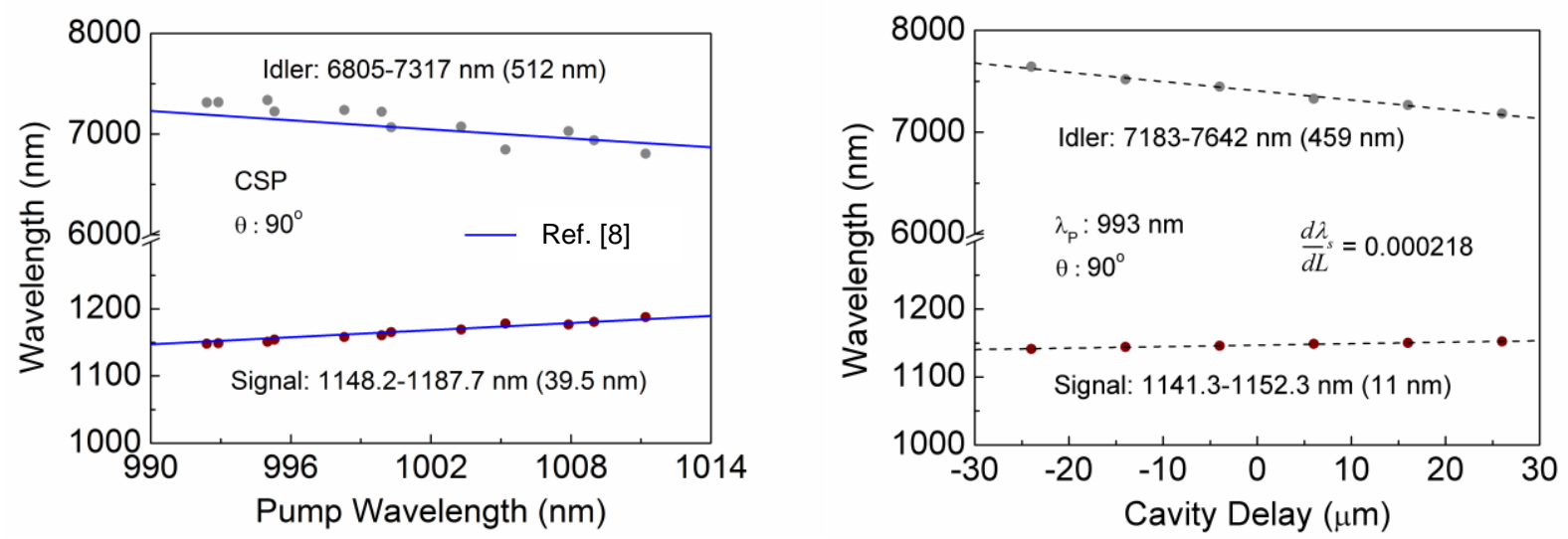

Figure 3. a) Pump tuning of the OPO compared to the Sellmeier predictions, ${ }^{8}$ b) Cavity delay tuning at normal incidence for a pump wavelength of $993 \mathrm{~nm}$.

Fig. 3(a) shows the experimental data for pump tuning, compared to the theoretical predictions in blue, calculated using the Sellmeier equations. ${ }^{8}$ As seen in Fig. 2(b), the relatively wide parametric gain bandwidth of CSP enables wavelength tuning by variation of cavity length. This is a convenient method for rapid tuning across a large idler bandwidth. An example of cavity delay tuning from this OPO at a pump wavelength of $993 \mathrm{~nm}$ and phase-matching angle of $\theta=90^{\circ}$ is shown in Fig. 3b). Using these methods in tandem, we are able to rapidly tune across $6805-7642 \mathrm{~nm}$ in the deep-IR.
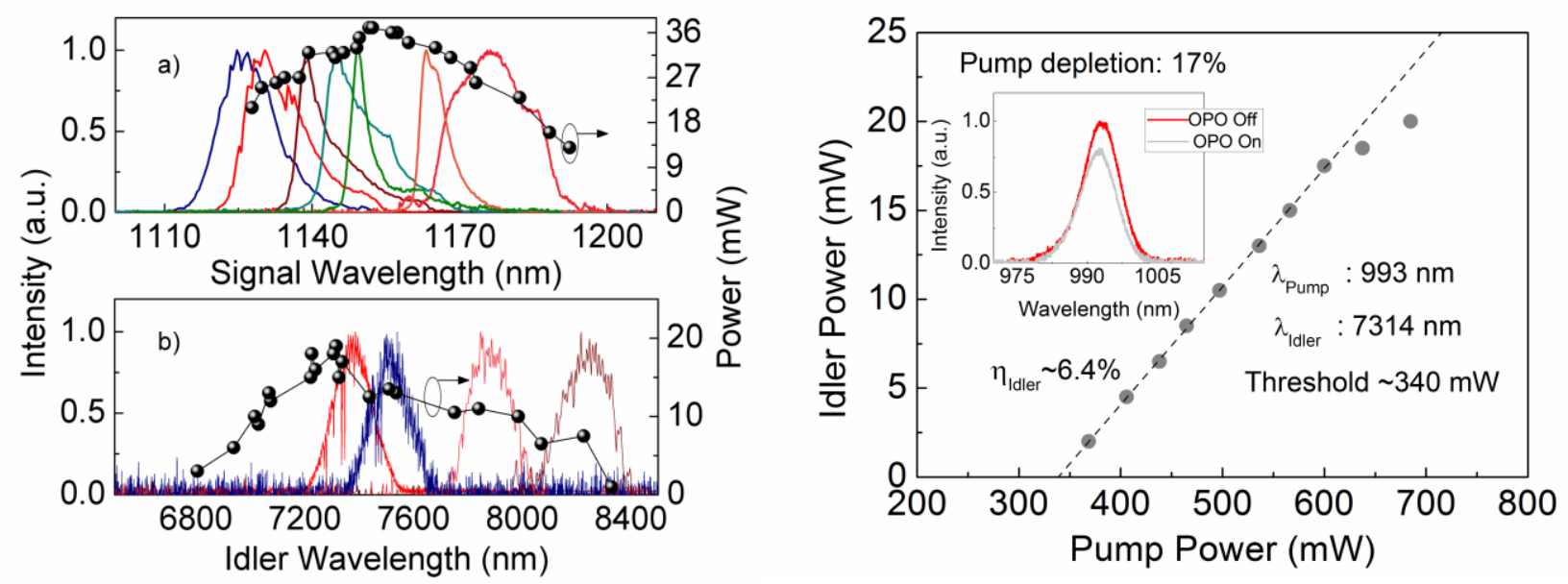

Figure 4. a) Signal, and b) idler power and spectra across the OPO tuning range, c) Power scaling of the idler extracted from the CSP femtosecond OPO. Inset: Input pump spectrum and depleted pump spectrum while the OPO is operating at maximum power.

As shown in Fig. 4(a), we extracted deep-IR idler average powers of $>10 \mathrm{~mW}$ across $>65 \%$ of the tuning range, with a maximum of $20 \mathrm{~mW}$ at $7314 \mathrm{~nm}$ and $>6 \mathrm{~mW}$ available up to $8227 \mathrm{~nm}$. This is equivalent to a peak idler quantum conversion efficiency of $21.3 \%$, and when the beam divergence and spectral bandwidth are considered, is equal to a spectral brightness of $\sim 6.68 \times 10^{20}$ photons s $\mathrm{sm}^{-1} \mathrm{sr}^{-1} 0.1 \% \mathrm{BW}^{-1}$. Furthermore, with a $5 \%$ output coupler in place, we measured signal powers up to $37 \mathrm{~mW}$ at $1152 \mathrm{~nm}$. Also plotted are the detailed spectral qualities of the signal and idler, characterised using two spectrum analysers for the near-IR and deep-IR, with resolutions of $0.7 \mathrm{~nm}$ and $\sim 4 \mathrm{~nm}$ respectively. The signal spectra were found to be highly asymmetric, containing an SPM-induced long-wavelength tail, as expected for a cavity with a large net positive GVD. In contrast, idler spectra were measured to be almost symmetric with evidence of atmospheric water absorption at shorter wavelengths in the form of deep modulations. We investigated idler power scaling of the OPO, using a high reflector in place of $\mathrm{M}_{8}$ to maximise the deep-IR power, with the results 
shown in Fig. 4(b). The pump power is corrected for a $\sim 83 \%$ transmission loss through M1. At a pump wavelength of $993 \mathrm{~nm}$, the OPO threshold was measured to be $\sim 340 \mathrm{~mW}$, and the idler slope conversion efficiency is $\sim 6.4 \%$, with a pump depletion of $\sim 17 \%$.
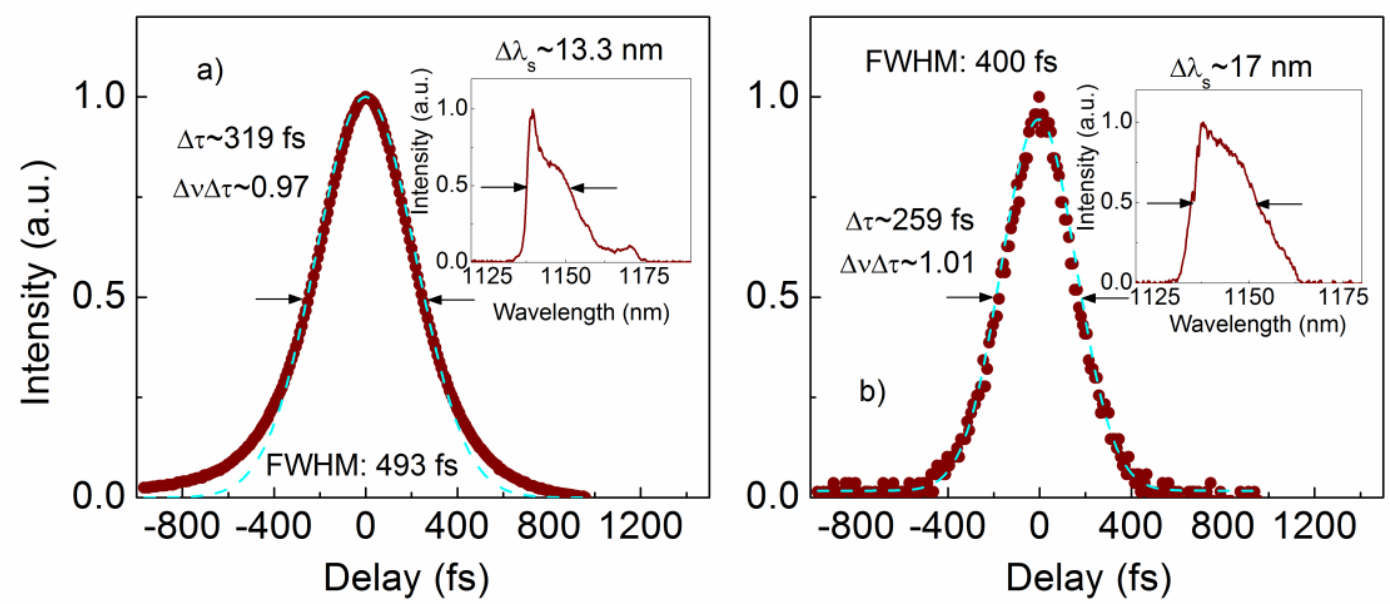

Figure 5. Signal intensity autocorrelations measured (a) at perfect cavity synchronisation, and (b) with the cavity detuned. Inset: simultaneously measured signal spectra.

The pulse duration was measured experimentally using an intensity autocorrelator, confirming strongly chirped pulses. Fig. 5 shows two signal autocorrelation profiles at (a) perfect synchronisation, and (b) when the cavity is slightly detuned, confirming durations of 319 and 259 fs, respectively. Also shown in the insets are the two corresponding spectra, with approximate FWHM bandwidths of $13.3 \mathrm{~nm}$ and $17 \mathrm{~nm}$, respectively. When the signal and pump pulses are not perfectly temporally overlapped, the reduced intracavity power induces a smaller nonlinear phase shift and results in a weaker pulse chirp.

\section{CONCLUSIONS}

In conclusion, we have demonstrated a high-spectral-brilliance, high-repetition-rate femtosecond source of coherent radiation for the deep-IR using a single frequency conversion stage pumped by a KLM Ti:sapphire laser. By exploiting the wide transparency window of CSP, we have been able to achieve successful operation of a deep-IR femtosecond OPO directly using a KLM Ti.sapphire laser, for the first time. In doing so, we have produced up to $20 \mathrm{~mW}$ of average power at $7314 \mathrm{~nm}$, equivalent to a spectral brightness of $6.68 \times 10^{20}$ photons s $\mathrm{mm}^{-1} \mathrm{sr}^{-1} 0.1 \% \mathrm{BW}^{-1}$, with up to $37 \mathrm{~mW}$ generated in the near-IR signal. We have used three separate tuning methods; type-I critical phase-matching, pump wavelength tuning, and cavity delay tuning, to generate coherent radiation across $1127-1192 \mathrm{~nm}$ and $6654-8373 \mathrm{~nm}$ $\left(1194-1503 \mathrm{~cm}^{-1}\right)$ in the signal and idler, respectively, with typical FWHM spectral bandwidths of $140-180 \mathrm{~nm}$ in the deep-IR. In particular, exploiting the tunable properties of the Ti:sapphire laser has permitted rapid tuning of the deep-IR idler wavelength over key spectral regions, including the amide III band, of great interest for medical imaging. The signal and idler beams exhibit excellent TEM $_{00}$ spatial quality and passive long-term power stability of $2.2 \%$ and $3.2 \%$ rms over 1 hour, respectively. Spectral and temporal characterisation of the signal has revealed chirped pulses with a time-bandwidth product, $\Delta v \Delta \tau \sim 1$. Both the pulse durations and $10 \mathrm{~dB}$ spectral bandwidths were observed to be broader at higher intracavity powers, indicating strong intracavity self-phase-modulation, which could be reduced using appropriate intracavity dispersion management. With high average power at a high repetition rate, this OPO represents an attractive source for low-noise spectroscopy, frequency comb generation and medical diagnostics in the 6.5-8.5 $\mu \mathrm{m}$ spectral region, using a simplified pumping method based on established ultrafast KLM Ti:sapphire laser technology. 


\section{REFERENCES}

[1] Bakker, J. M., Mac Aleese, L., Meijer, G. and von Helden, G., "Fingerprint IR spectroscopy to probe amino acid conformations in the gas phase," Phys. Rev. Lett. 91(20), 203003 (2003).

[2] Bellamy, L. J., [The Infra-red Spectra of Complex Molecules], Springer Netherlands, 231-262 (1975).

[3] Mille, L. M., and Smith, R. J., "Synchrotrons versus globars, point-detectors versus focal plane arrays: Selecting the best source and detector for specific infrared microspectroscopy and imaging applications," Vib. Spectrosc. 38(1), 237-240, (2005).

[4] Petersen, C. R., Møller, U., Kunbat, I., Zhou, B., Dupont, S., Ramsay, J., Benson, T., Sujecki, S., Abdel-Moneim, N., Tang, Z., and Furniss, D., "Mid-infrared supercontinuum covering the 1.4-13.3 $\mu \mathrm{m}$ molecular finger-print region using ultra-high NA chalcogenide step-index fibre," Nat. Photonics 8(11), 830-834, (2014).

[5] Dumas, P., Polack, F., Lagarde, B., Chubar, O., Giorgetta, J. L., and Lefrançois, S., "Synchrotron infrared microscopy at the French Synchrotron Facility SOLEIL," Infrared Phys. Techn. 49(1), 152-160, (2006).

[6] Faist, J., [Quantum cascade lasers], UK: OUP Oxford, (2013).

[7] Ebrahim-Zadeh, M., and Sorokina, I. T., [Mid-infrared Coherent Sources and Applications], Springer, (2008).

[8] Zawilski, K. T., Schunemann, P. G., Pollak, T. M., Zelmon, D. E., Fernelius, N. C., and Hopkins, F. K., "Growth and characterization of large CdSiP2 single crystals," J. Cryst. Growth 312(8), 1127-1132, (2010).

[9] Chaitanya Kumar, S., Schunemann, P. G., Zawilski, K. T., and Ebrahim-Zadeh, M., "Advances in ultrafast optical parametric sources for the mid-infrared based on CdSiP2,” J. Opt. Soc. Am. B 33(11), D44-D56, (2016).

[10] Zhang, Z., Reid, D. T., Chaitanya Kumar., S., Ebrahim-Zadeh, M., Schunemann, P. G., Zawilski, K. T. and Howle, C. R., "Femtosecond-laser pumped $\mathrm{CdSiP}_{2}$ optical parametric oscillator producing $100 \mathrm{MHz}$ pulses centered at 6.2 $\mu \mathrm{m}$, , Opt. Lett. 38(23), 5110-5113, (2013).

[11] Chaitanya Kumar, S., Krauth, J., Steinmann A., Zawilski, K. T., Schunemann, P. G., Giessen, H. and EbrahimZadeh, M., "High-power femtosecond mid-infrared optical parametric oscillator at $7 \mu \mathrm{m}$ based on $\mathrm{CdSiP}_{2}$," Opt. Lett. 40(7), 1398-1401, (2015).

[12] Ramaiah-Badarla, V., Chaitanya Kumar, S., Esteban-Martin, A., Devi, K., Zawilski, K. T., Schunemann, P. G., and Ebrahim-Zadeh, M., "Ti:sapphire-pumped deep-infrared femtosecond optical parametric oscillator based on CdSiP2,” Opt. Lett. 41(8), 1708-1711, (2016).

[13] Chaitanya Kumar, S., Esteban-Martin, A., Santana, A., Zawilski, K. T., Schunemann, P. G., and Ebrahim-Zadeh, M., "Pump-tuned deep-infrared femtosecond optical parametric oscillator across 6-7 $\mu \mathrm{m}$ based on $\mathrm{CdSiP}_{2}$," Opt. Lett. 41(14), 3355-3358, (2016). 